\title{
Asymptotic decay estimate of solutions to the generalized damped Bq equation
}

Yinxia Wang*

\section{*Correspondence:}

yinxia117@126.com

School of Mathematics and

Information Sciences, North China

University of Water Resources and

Electric Power, Zhengzhou, 450011,

China

\begin{abstract}
In this paper, we investigate the Cauchy problem for the generalized damped Bq equation. By applying fixed point theorem, we prove the global existence and asymptotic decay estimate of solutions for all space dimensions $n \geq 1$ provided that the initial value is suitably small.
\end{abstract}

MSC: 35L30; 35L75

Keywords: generalized damped Boussinesq equation; fixed point theorem; decay estimate

\section{Introduction}

We study asymptotic decay estimate of solution to the Cauchy problem for the generalized damped Bq equation:

$$
u_{t t}-\Delta u-2 b \Delta u_{t}+\alpha \Delta^{2} u=\Delta f(u)
$$

with the initial value

$$
t=0: \quad u=u_{0}(x), \quad u_{t}=u_{1}(x) .
$$

Here $u=u(x, t)$ is the unknown function of $x=\left(x_{1}, \ldots, x_{n}\right) \in \mathbb{R}^{n}$ and $t>0, b>0$ and $\alpha>0$ are constants. The nonlinear term $f(u)$ is a given smooth function of $u \in \mathbb{R}$ satisfying $f(u)=O\left(u^{2}\right)$ for $u \rightarrow 0$.

It is well known that the equation (called the classical Bq equation)

$$
u_{t t}+\alpha \Delta^{2} u-\Delta u=\beta \Delta\left(u^{2}\right)
$$

was derived by Boussinesq [1] in 1872 to describe shallow water waves, where $u(x, t)$ is an elevation of the free surface of fluid and the constant coefficients $\alpha$ and $\beta$ depend on the depth of fluid and the characteristic speed of long waves. It is interesting to note that this equation governs nonlinear string oscillations as well. For (1.3) and the generalized Bq equation, there are lots of important results (see [2-5]).

Equation (1.3) takes into account dispersion and nonlinearity, but in real processes viscosity also plays an important role. Varlamov considered the following damped Boussinesq equation (see [6-8]):

$$
u_{t t}-\Delta u-2 b \Delta u_{t}+\alpha \Delta^{2} u=\beta \Delta\left(u^{2}\right),
$$

(0) 2013 Wang; licensee Springer. This is an Open Access article distributed under the terms of the Creative Commons Attribution License (http://creativecommons.org/licenses/by/2.0), which permits unrestricted use, distribution, and reproduction in any medium, provided the original work is properly cited. 
where $b>0$ and $\alpha>0$ are constants. A classical solution to the Cauchy problem for (1.4) with small initial data was constructed by means of the application of both spectral and perturbation theories. Large time asymptotics of this solution was also obtained (see [6]). Varlamov [7] investigated the Cauchy problem for (1.4). For the cases of one, two and three space dimensions local in time existence and uniqueness of a solution is proved. The authors also showed that for discontinuous initial perturbations this solution is infinitely differentiable with respect to time $t$ and space co-ordinates for $t>0$ on a bounded time interval. Varlamov [8] considered the Cauchy problem for (1.4) with small initial data in two space dimensions. Existence and uniqueness of the classical solution was proved and the solution was constructed in the form of a series. The major term of its long-time asymptotics is calculated explicitly and a uniform in space estimate of the residual term was given.

The main purpose of this paper is to establish the following asymptotic decay estimate of solutions to (1.1), (1.2) for $n \geq 1$ :

$$
\left\|\partial_{x}^{k} u(t)\right\|_{H^{s+2-k}} \leq C E_{0}(1+t)^{-\frac{n}{4}-\frac{k}{2}-\frac{1}{2}}
$$

for $0 \leq k \leq s+2$ and $s \geq \max \left\{0,\left[\frac{n}{2}\right]-1\right\}$. Here $E_{0}:=\left\|u_{0}\right\|_{\dot{W}^{-1,1}}+\left\|u_{1}\right\|_{\dot{W}^{-2,1}}+\left\|u_{0}\right\|_{H^{s+2}}+$ $\left\|u_{1}\right\|_{H^{s}}$.

Decay estimate of solutions to hyperbolic-type equations has been investigated by many authors. We refer to $[9,10]$ for hyperbolic equations, to [11-15] for damped wave equation and to [16-19] for various aspects of dissipation of the plate equation.

The paper is organized as follows. In Section 2 we derive the solution formula of our semi-linear problem. We study the decay property of the solution operators appearing in the solution formula in Section 3. In Section 4, asymptotic decay estimate of solutions to the cauchy problem (1.1), (1.2) is established by applying fixed point theorem.

Notations We give some notations which are used in this paper. Let $\mathcal{F}[u]$ denote the Fourier transform of $u$ defined by

$$
\hat{u}(\xi)=\mathcal{F}[u]=\int_{\mathbb{R}^{n}} e^{-i \xi \cdot x} u(x) d x,
$$

and we denote its inverse transform by $\mathcal{F}^{-1}$.

For $1 \leq p \leq \infty, L^{p}=L^{p}\left(\mathbb{R}^{n}\right)$ denotes the usual Lebesgue space with the norm $\|\cdot\|_{L^{p}}$. The usual Sobolev space of $s$ is defined by $W^{s, p}=(I-\Delta)^{-\frac{s}{2}} L^{p}$ with the norm $\|f\|_{W^{s, p}}=$ $\left\|(I-\Delta)^{\frac{s}{2}} f\right\|_{L^{p}}$; the homogeneous Sobolev space of $s$ is defined by $\dot{W}^{s, p}=(-\Delta)^{-\frac{s}{2}} L^{p}$ with the norm $\|f\|_{\dot{W}^{s, p}}=\left\|(-\Delta)^{\frac{s}{2}} f\right\|_{L^{p}}$; especially $H^{s}=W^{s, 2}, \dot{H}^{s}=\dot{W}^{s, 2}$. Moreover, we know that $W^{s, p}=L^{p} \cap \dot{W}^{s, p}$ for $s \geq 0$.

Finally, in this paper, we denote every positive constant by the same symbol $C$ or $c$ without confusion. [.] is the Gauss symbol.

\section{Solution formula}

The aim of this section is to derive the solution formula for the problem (1.1), (1.2). We first investigate the linearized equation of (1.1).

$$
u_{t t}-\Delta u-2 b \Delta u_{t}+\alpha \Delta^{2} u=0
$$


with the initial data in (1.2). Taking the Fourier transform, we have

$$
\hat{u}_{t t}+2 b|\xi|^{2} \hat{u}_{t}+\left(|\xi|^{2}+\alpha|\xi|^{4}\right) \hat{u}=0
$$

The corresponding initial values are given as

$$
t=0: \quad \hat{u}=\hat{u}_{0}(\xi), \quad \hat{u}_{t}=\hat{u}_{1}(\xi) .
$$

The characteristic equation of (2.1) is

$$
\lambda^{2}+2 b|\xi|^{2} \lambda+|\xi|^{2}+\alpha|\xi|^{4}=0
$$

Let $\lambda=\lambda_{ \pm}(\xi)$ be the corresponding eigenvalues of (2.3), we obtain

$$
\lambda_{ \pm}(\xi)=-b|\xi|^{2} \pm|\xi| \sqrt{\left(b^{2}-\alpha\right)|\xi|^{2}-1} .
$$

The solution to the problem (2.1)-(2.2) is given in the form

$$
\hat{u}(\xi, t)=\hat{G}(\xi, t) \hat{u}_{1}(\xi)+\hat{H}(\xi, t) \hat{u}_{0}(\xi),
$$

where

$$
\hat{G}(\xi, t)=\frac{1}{\lambda_{+}(\xi)-\lambda_{-}(\xi)}\left(e^{\lambda_{+}(\xi) t}-e^{\lambda_{-}(\xi) t}\right)
$$

and

$$
\hat{H}(\xi, t)=\frac{1}{\lambda_{+}(\xi)-\lambda_{-}(\xi)}\left(\lambda_{+}(\xi) e^{\lambda_{-}(\xi) t}-\lambda_{-}(\xi) e^{\lambda_{+}(\xi) t}\right) .
$$

We define $G(x, t)$ and $H(x, t)$ by

$$
G(x, t)=\mathcal{F}^{-1}[\hat{G}(\xi, t)](x)
$$

and

$$
H(x, t)=\mathcal{F}^{-1}[\hat{H}(\xi, t)](x),
$$

respectively, where $\mathcal{F}^{-1}$ denotes the inverse Fourier transform. Then, applying $\mathcal{F}^{-1}$ to (2.5), we obtain

$$
u(t)=G(t) * u_{1}+H(t) * u_{0} .
$$

By the Duhamel principle, we obtain the solution formula to (1.1), (1.2)

$$
u(t)=G(t) * u_{1}+H(t) * u_{0}+\int_{0}^{t} G(t-\tau) * \Delta f(u)(\tau) d \tau
$$




\section{Decay property}

The aim of this section is to establish decay estimates of the solution operators $G(t)$ and $H(t)$ appearing in the solution formula (2.10).

Lemma 3.1 The solution of the problem (2.1), (2.2) satisfies

$$
\left(|\xi|^{2}+|\xi|^{4}\right)|\hat{u}(\xi, t)|^{2}+\left|\hat{u}_{t}(\xi, t)\right|^{2} \leq C e^{-c|\xi|^{2} t}\left(\left(|\xi|^{2}+|\xi|^{4}\right)\left|\hat{u}_{0}(\xi)\right|^{2}+\left|\hat{u}_{1}(\xi)\right|^{2}\right)
$$

for $\xi \in \mathbb{R}^{n}$ and $t \geq 0$.

Proof Multiplying (2.1) by $\overline{\hat{u}}_{t}$ and taking the real part yields

$$
\frac{1}{2} \frac{d}{d t}\left\{\left|\hat{u}_{t}\right|^{2}+|\xi|^{2}|\hat{u}|^{2}+\alpha|\xi|^{4}|\hat{u}|^{2}\right\}+2 b|\xi|^{2}\left|\hat{u}_{t}\right|^{2}=0
$$

Multiplying (2.1) by $\overline{\hat{u}}$ and taking the real part, we obtain

$$
\frac{1}{2} \frac{d}{d t}\left\{2 b|\xi|^{2}|\hat{u}|^{2}+2 \operatorname{Re}\left(\hat{u}_{t} \overline{\hat{u}}\right)\right\}+|\xi|^{2}|\hat{u}|^{2}+\alpha|\xi|^{4}|\hat{u}|^{2}-\left|\hat{u}_{t}\right|^{2}=0
$$

Multiplying both sides of (3.3) by $b|\xi|^{2}$ and summing up the resulting equation and (3.2) yield

$$
\frac{d}{d t} E+F=0
$$

where

$$
E=\frac{1}{2}\left|\hat{u}_{t}\right|^{2}+\frac{1}{2}|\xi|^{2}|\hat{u}|^{2}+\frac{\alpha}{2}|\xi|^{4}|\hat{u}|^{2}+b^{2}|\xi|^{4}|\hat{u}|^{2}+b|\xi|^{2} \operatorname{Re}\left(\hat{u}_{t} \overline{\hat{u}}\right)
$$

and

$$
F=b|\xi|^{2}\left|\hat{u}_{t}\right|^{2}+b|\xi|^{4}|\hat{u}|^{2}+b \alpha|\xi|^{6}|\hat{u}|^{2} .
$$

A simple computation implies that

$$
C E_{0} \leq E \leq C E_{0}
$$

where

$$
E_{0}=\left(|\xi|^{2}+|\xi|^{4}\right)|\hat{u}|^{2}+\left|\hat{u}_{t}\right|^{2} .
$$

Note that

$$
F \geq c|\xi|^{2} E_{0}
$$

It follows from (3.5) that

$$
F \geq c|\xi|^{2} E .
$$


Using (3.4) and (3.6), we get

$$
\frac{d}{d t} E+c|\xi|^{2} E \leq 0 .
$$

Thus

$$
E(\xi, t) \leq e^{-c|\xi|^{2} t} E(\xi, 0)
$$

which together with (3.5) proves the desired estimates (3.1). Then we have completed the proof of the lemma.

Lemma 3.2 Let $\hat{G}(\xi, t)$ and $\hat{H}(\xi, t)$ be the fundamental solutions of the linear equation of (1.1) in the Fourier space, which are given in (2.6) and (2.7), respectively. Then we have the estimates

$$
\left(|\xi|^{2}+|\xi|^{4}\right)|\hat{G}(\xi, t)|^{2}+\left|\hat{G}_{t}(\xi, t)\right|^{2} \leq C e^{-c|\xi|^{2} t}
$$

and

$$
\left(|\xi|^{2}+|\xi|^{4}\right)|\hat{H}(\xi, t)|^{2}+\left|\hat{H}_{t}(\xi, t)\right|^{2} \leq C\left(|\xi|^{2}+|\xi|^{4}\right) e^{-c|\xi|^{2} t}
$$

for $\xi \in \mathbb{R}^{n}$ and $t \geq 0$.

Proof If $\hat{u}_{0}(\xi)=0$, from (2.5), we obtain

$$
\hat{u}(\xi, t)=\hat{G}(\xi, t) \hat{u}_{1}(\xi), \quad \hat{u}_{t}(\xi, t)=\hat{G}_{t}(\xi, t) \hat{u}_{1}(\xi) .
$$

Substituting the equalities into (3.1) with $\hat{u}_{0}(\xi)=0$, we get (3.7).

In what follows, we consider $\hat{u}_{1}(\xi)=0$, it follows from $(2.5)$ that

$$
\hat{u}(\xi, t)=\hat{H}(\xi, t) \hat{u}_{0}(\xi), \quad \hat{u}_{t}(\xi, t)=\hat{H}_{t}(\xi, t) \hat{u}_{0}(\xi)
$$

Substituting the equalities into (3.1) with $\hat{u}_{1}(\xi)=0$, we get the desired estimate (3.8). The lemma is proved.

Lemma 3.3 Let $G(x, t)$ and $H(x, t)$ be the fundamental solutions of the linear equation of (1.1), which are given in (2.8) and (2.9), respectively. Then, for $k \geq 0$ and $j \geq 0$, we have

$$
\begin{aligned}
& \left\|\partial_{x}^{k} G(t) * \phi\right\|_{L^{2}} \leq C(1+t)^{-\left(\frac{n}{4}+\frac{k}{2}+\frac{j}{2}-\frac{1}{2}\right)}\|\phi\|_{\dot{W}^{-j, 1}}+C e^{-c t}\left\|\partial_{x}^{(k-2)_{+}} \phi\right\|_{L^{2}}, \\
& \left\|\partial_{x}^{k} H(t) * \phi\right\|_{L^{2}} \leq C(1+t)^{-\left(\frac{n}{4}+\frac{k}{2}+\frac{j}{2}\right)}\|\phi\|_{\dot{W}^{-j, 1}}+C e^{-c t}\left\|\partial_{x}^{k} \phi\right\|_{L^{2}}, \\
& \left\|\partial_{x}^{k} G_{t}(t) * \phi\right\|_{L^{2}} \leq C(1+t)^{-\left(\frac{n}{4}+\frac{k}{2}+\frac{j}{2}\right)}\|\phi\|_{\dot{W}^{-j, 1}}+C e^{-c t}\left\|\partial_{x}^{k} \phi\right\|_{L^{2}}, \\
& \left\|\partial_{x}^{k} H_{t}(t) * \phi\right\|_{L^{2}} \leq C(1+t)^{-\left(\frac{n}{4}+\frac{k}{2}+\frac{j}{2}+\frac{1}{2}\right)}\|\phi\|_{\dot{W}^{-j, 1}}+C e^{-c t}\left\|\partial_{x}^{(k+2)} \phi\right\|_{L^{2}}
\end{aligned}
$$


and

$$
\begin{aligned}
& \left\|\partial_{x}^{k} G(t) * \Delta g\right\|_{L^{2}} \leq C(1+t)^{-\left(\frac{n}{4}+\frac{k}{2}+\frac{1}{2}\right)}\|g\|_{L^{1}}+C e^{-c t}\left\|\partial_{x}^{k} g\right\|_{L^{2}}, \\
& \left\|\partial_{x}^{k} G_{t}(t) * \Delta g\right\|_{L^{2}} \leq C(1+t)^{-\left(\frac{n}{4}+\frac{k}{2}+1\right)}\|g\|_{L^{1}}+C e^{-c t}\left\|\partial_{x}^{(k+2)} g\right\|_{L^{2}},
\end{aligned}
$$

where $(k-2)_{+}=\max \{0, k-2\}$ in $(3.9)$.

Proof By the Plancherel theorem and (3.7), the Hausdorff-Young inequality, we obtain

$$
\begin{aligned}
\left\|\partial_{x}^{k} G(t) * \phi\right\|_{L^{2}}^{2}= & \int_{|\xi| \leq R_{0}}|\xi|^{2 k}|\hat{G}(\xi, t)|^{2}|\hat{\phi}(\xi)|^{2} d \xi \\
& +\int_{|\xi| \geq R_{0}}|\xi|^{2 k}|\hat{G}(\xi, t)|^{2}|\hat{\phi}(\xi)|^{2} d \xi \\
\leq & \int_{|\xi| \leq R_{0}}|\xi|^{2 k-2} e^{-c|\xi|^{2} t}|\hat{\phi}(\xi)|^{2} d \xi \\
& +C e^{-c t} \int_{|\xi| \geq R_{0}}|\xi|^{2 k}\left(|\xi|^{2}+|\xi|^{4}\right)^{-1}|\hat{\phi}(\xi)|^{2} d \xi \\
\leq & \int_{|\xi| \leq R_{0}}|\xi|^{2 k-2+2 j} e^{-c|\xi|^{2} t}|\xi|^{-2 j}|\hat{\phi}(\xi)|^{2} d \xi+C e^{-c t}\left\|\partial_{x}^{(k-2)_{+}} \phi\right\|_{L^{2}}^{2} \\
\leq & C\left\||\xi|^{-j} \hat{\phi}(\xi)\right\|_{L^{\infty}}^{2} \int_{|\xi| \leq R_{0}}|\xi|^{2 k-2+2 j} e^{-c|\xi|^{2} t} d \xi+C e^{-c t}\left\|\partial_{x}^{(k-2)_{+}} \phi\right\|_{L^{2}}^{2} \\
\leq & C(1+t)^{-\left(\frac{n}{2}+k+j-1\right)}\left\|(-\Delta)^{-\frac{j}{2}} \phi\right\|_{L^{1}}^{2}+C e^{-c t}\left\|\partial_{x}^{(k-2)_{+}} \phi\right\|_{L^{2}}^{2},
\end{aligned}
$$

where $(k-2)_{+}=\max \{0, k-2\}$ and $R_{0}$ is a small positive constant. Thus (3.9) follows.

Similarly, using (3.7) and (3.8), respectively, we can prove (3.10)-(3.12).

In what follows, we prove (3.13). By the Plancherel theorem, (3.7) and the HausdorffYoung inequality, we have

$$
\begin{aligned}
\left\|\partial_{x}^{k} G(t) * \Delta g\right\|_{L^{2}}^{2}= & \int_{|\xi| \leq R_{0}}|\xi|^{2|k|}|\hat{G}(\xi, t)|^{2}|\xi|^{4}|\hat{g}(\xi)|^{2} d \xi \\
& +\int_{|\xi| \geq R_{0}}|\xi|^{2 k}|\hat{G}(\xi, t)|^{2}|\xi|^{4}|\hat{g}(\xi)|^{2} d \xi \\
\leq & \int_{|\xi| \leq R_{0}}|\xi|^{2 k+2} e^{-c|\xi|^{2} t}|\hat{g}(\xi)|^{2} d \xi+C e^{-c t} \int_{|\xi| \geq R_{0}}|\xi|^{2 k}|\hat{g}(\xi)|^{2} d \xi \\
\leq & C\|\hat{g}(\xi)\|_{L^{\infty}}^{2} \int_{|\xi| \leq R_{0}}|\xi|^{2 k+2} e^{-c|\xi|^{2} t} d \xi+C e^{-c t}\left\|\partial_{x}^{k} g\right\|_{L^{2}}^{2} \\
\leq & C(1+t)^{-\left(\frac{n}{2}+k+1\right)}\|g\|_{L^{1}}^{2}+C e^{-c t}\left\|\partial_{x}^{k} g\right\|_{L^{2}}^{2},
\end{aligned}
$$

where $R_{0}$ is a small positive constant. Thus (3.13) follows. Similarly, we can prove (3.14). Thus we have completed the proof of the lemma.

\section{Asymptotic decay of solutions to (1.1), (1.2)}

The purpose of this section is to prove asymptotic decay of solutions to the Cauchy problem (1.1), (1.2). We need the following lemma, which comes from [20] (see also [21]). 
Lemma 4.1 Assume that $f=f(v)$ is a smooth function. Suppose that $f(v)=O\left(|v|^{1+\theta}\right)(\theta \geq 1$ is an integer) when $|v| \leq v_{0}$. Then, for integer $m \geq 0$, if $v, w \in W^{m, q}\left(\mathbb{R}^{n}\right) \cap L^{p}\left(\mathbb{R}^{n}\right) \cap L^{\infty}\left(\mathbb{R}^{n}\right)$ and $\|v\|_{L^{\infty}} \leq v_{0},\|w\|_{L^{\infty}} \leq v_{0}$, then $f(v)-f(w) \in W^{m, r}\left(\mathbb{R}^{n}\right)$. Furthermore, the following inequalities hold:

$$
\left\|\partial_{x}^{m} f(v)\right\|_{L^{r}} \leq C\|v\|_{L^{p}}\left\|\partial_{x}^{m} v\right\|_{L^{q}}\|v\|_{L^{\infty}}^{\theta-1}
$$

and

$$
\begin{aligned}
\left\|\partial_{x}^{m}(f(v)-f(w))\right\|_{L^{r}} \leq & C\left\{\left(\left\|\partial_{x}^{m} v\right\|_{L^{q}}+\left\|\partial_{x}^{m} w\right\|_{L^{q}}\right)\|v-w\|_{L^{p}}\right. \\
& \left.+\left(\|v\|_{L^{p}}+\|w\|_{L^{p}}\right)\left\|\partial_{x}^{m}(v-w)\right\|_{L^{q}}\right\}\left(\|v\|_{L^{\infty}}+\|w\|_{L^{\infty}}\right)^{\theta-1},
\end{aligned}
$$

where $\frac{1}{r}=\frac{1}{p}+\frac{1}{q}, 1 \leq p, q, r \leq+\infty$.

In the previous section, we showed the decay estimates for the solution operators. With this preparation, we can prove the global existence and asymptotic decay of solutions to the integral equation (2.11) and hence to the problem (1.1), (1.2). So we define the following solution space:

$$
X=\left\{u \in C\left([0, \infty) ; H^{s+2}\left(\mathbb{R}^{n}\right)\right) \cap C^{1}\left([0, \infty) ; H^{s}\left(\mathbb{R}^{n}\right)\right):\|u\|_{X}<\infty\right\},
$$

where

$$
\|u\|_{X}=\sup _{t \geq 0}\left\{\sum_{k \leq s+2}(1+t)^{\frac{n}{4}+\frac{k}{2}+\frac{1}{2}}\left\|\partial_{x}^{k} u(t)\right\|_{L^{2}}+\sum_{l \leq s}(1+t)^{\frac{n}{4}+\frac{l}{2}+1}\left\|\partial_{x}^{l} u_{t}(t)\right\|_{L^{2}}\right\} .
$$

For $R>0$, we define

$$
X_{R}=\left\{u \in X:\|u\|_{X} \leq R\right\} .
$$

Using Gagliardo-Nirenberg inequality, we obtain

$$
\|u(t)\|_{L^{\infty}} \leq C\left\|\partial_{x}^{s_{0}} u(t)\right\|_{L^{2}}^{\theta}\|u(t)\|_{L^{2}}^{1-\theta} \leq C R(1+t)^{-\frac{n}{4}-\frac{1}{2}}
$$

where

$$
s_{0}=\left[\frac{n}{2}\right]+1, \quad \theta=\frac{n}{2 s_{0}}, \quad s_{0} \leq s+2\left(\text { i.e., } s \geq\left[\frac{n}{2}\right]-1\right) .
$$

Theorem 4.1 Assume that $u_{0} \in H^{s+2}\left(\mathbb{R}^{n}\right) \cap \dot{W}^{-1,1}\left(\mathbb{R}^{n}\right), u_{1} \in H^{s}\left(\mathbb{R}^{n}\right) \cap \dot{W}^{-2,1}\left(\mathbb{R}^{n}\right)(s \geq$ $\left.\max \left\{0,\left[\frac{n}{2}\right]-1\right\}\right)$. Put

$$
E_{0}:=\left\|u_{0}\right\|_{\dot{W}^{-1,1}}+\left\|u_{1}\right\|_{\dot{W}^{-2,1}}+\left\|u_{0}\right\|_{H^{s+2}}+\left\|u_{1}\right\|_{H^{s}}
$$

If $E_{0}$ is suitably small, the Cauchy problem (1.1), (1.2) has a unique global classical solution $u(x, t)$ satisfying

$$
u \in C^{0}\left([0, \infty) ; H^{s+2}\left(\mathbb{R}^{n}\right)\right) C^{1}\left([0, \infty) ; H^{s}\left(\mathbb{R}^{n}\right)\right) .
$$


Moreover, the solution satisfies the decay estimate

$$
\left\|\partial_{x}^{k} u(t)\right\|_{L^{2}} \leq C E_{0}(1+t)^{-\left(\frac{n}{4}+\frac{k}{2}+\frac{1}{2}\right)}
$$

and

$$
\left\|\partial_{x}^{l} u_{t}(t)\right\|_{L^{2}} \leq C E_{0}(1+t)^{-\left(\frac{n}{4}+\frac{l}{2}+1\right)}
$$

for $k \leq s+2$ and $l \leq s$.

Proof Define the mapping

$$
\mathbb{T}(u)=G(t) * u_{1}+H(t) * u_{0}+\int_{0}^{t} G(t-\tau) * \Delta f(u(\tau)) d \tau .
$$

Using (3.9) and (3.10) with $j=2,1$, respectively, (3.13), Lemma 4.1 and (4.1), for $k \leq s+2$, we obtain

$$
\begin{aligned}
\left\|\partial_{x}^{k} \mathbb{T}(u)\right\|_{L^{2}} \leq & C\left\|\partial_{x}^{k} G(t) * u_{1}\right\|_{L^{2}}+C\left\|\partial_{x}^{k} H(t) * u_{0}\right\|_{L^{2}} \\
& +C \int_{0}^{t}\left\|\partial_{x}^{k} G(t-\tau) * \Delta f(u(\tau))\right\|_{L^{2}} d \tau \\
\leq & C(1+t)^{-\left(\frac{n}{4}+\frac{k}{2}+\frac{1}{2}\right)}\left(\left\|u_{0}\right\|_{\dot{W}^{-1,1}}+\left\|u_{1}\right\|_{\dot{W}^{-2,1}}\right)+C e^{-c t}\left(\left\|u_{0}\right\|_{H^{s+2}}+\left\|u_{1}\right\|_{H^{s}}\right) \\
& +C \int_{0}^{\frac{t}{2}}(1+t-\tau)^{-\left(\frac{n}{4}+\frac{k}{2}+\frac{1}{2}\right)}\|f(u)\|_{L^{1}} d \tau \\
& +C \int_{\frac{t}{2}}^{t}(1+t-\tau)^{-\left(\frac{n}{4}+\frac{1}{2}\right)}\left\|\partial_{x}^{k} f(u)\right\|_{L^{1}} d \tau \\
& +C \int_{0}^{t} e^{-c(t-\tau)}\left\|\partial_{x}^{k} f(u)\right\|_{L^{2}} d \tau \\
\leq & C(1+t)^{-\left(\frac{n}{4}+\frac{k}{2}+\frac{1}{2}\right)}\left(\left\|u_{0}\right\|_{\dot{W}^{-1,1}}+\left\|u_{1}\right\|_{\dot{W}^{-2,1}}\right)+C e^{-c t}\left(\left\|u_{0}\right\|_{H^{s+2}}+\left\|u_{1}\right\|_{H^{s}}\right) \\
& +C \int_{0}^{\frac{t}{2}}(1+t-\tau)^{-\left(\frac{n}{4}+\frac{k}{2}+\frac{1}{2}\right)}\|u\|_{L^{2}}^{2} d \tau \\
& +C \int_{\frac{t}{2}}^{t}(1+t-\tau)^{-\left(\frac{n}{4}+\frac{1}{2}\right)}\|u\|_{L^{2}}\left\|\partial_{x}^{k} u\right\|_{L^{2}} d \tau \\
& +C \int_{0}^{t} e^{-c(t-\tau)}\left\|\partial_{x}^{k} u\right\|_{L^{2}}\|u\|_{L^{\infty}} d \tau \\
\leq & C(1+t)^{-\left(\frac{n}{4}+\frac{k}{2}+\frac{1}{2}\right)}\left(\left\|u_{0}\right\|_{\dot{W}^{-1,1}}+\left\|u_{1}\right\|_{\dot{W}^{-2,1}}\right)+C e^{-c t}\left(\left\|u_{0}\right\|_{H^{s+2}}+\left\|u_{1}\right\|_{H^{s}}\right) \\
& +C R^{2} \int_{0}^{\frac{t}{2}}(1+t-\tau)^{-\left(\frac{n}{4}+\frac{k}{2}+\frac{1}{2}\right)}(1+\tau)^{-\left(\frac{n}{2}+1\right)} d \tau \\
& +C R^{2} \int_{\frac{t}{2}}^{t}(1+t-\tau)^{-\left(\frac{n}{4}+\frac{1}{2}\right)}(1+\tau)^{-\left(\frac{n}{2}+\frac{k}{2}+1\right)} d \tau \\
& +t)^{-\left(\frac{n}{4}+\frac{k}{2}+\frac{1}{2}\right)}\left\{\left(\left\|u_{0}\right\|_{\dot{W}^{-1,1}}+\left\|u_{1}\right\|_{\dot{W}^{-2,1}}+\left\|u_{0}\right\|_{H^{s+2}}+\left\|u_{1}\right\|_{H^{s}}\right)+R^{2}\right\} \\
& \\
& \\
& \\
& \\
& \\
& \\
&
\end{aligned}
$$


Thus

$$
(1+t)^{\frac{n}{4}+\frac{k}{2}+\frac{1}{2}}\left\|\partial_{x}^{k} \mathbb{T}(u)\right\|_{L^{2}} \leq C E_{0}+C R^{2} .
$$

It follows from (4.4) that

$$
\mathbb{T}(u)_{t}=G_{t}(t) * u_{1}+H_{t}(t) * u_{0}+\int_{0}^{t} G_{t}(t-\tau) * \Delta f(u(\tau)) d \tau .
$$

By using (3.11) and (3.12) with $j=2,1$, respectively, (3.14) Lemma 4.1 and (4.1), for $l \leq s$, we have

$$
\begin{aligned}
\left\|\partial_{x}^{l} \mathbb{T}(u)_{t}\right\|_{L^{2}} \leq & C\left\|\partial_{x}^{l} G_{t}(t) * u_{1}\right\|_{L^{2}}+C\left\|\partial_{x}^{l} H_{t}(t) * u_{0}\right\|_{L^{2}} \\
& +C \int_{0}^{t}\left\|\partial_{x}^{l} G_{t}(t-\tau) * \Delta f(u(\tau))\right\|_{L^{2}} d \tau \\
\leq & C(1+t)^{-\left(\frac{n}{4}+\frac{l}{2}+1\right)}\left(\left\|u_{0}\right\|_{\dot{W}^{-1,1}}+\left\|u_{1}\right\|_{\dot{W}^{-2,1}}\right)+C e^{-c t}\left(\left\|u_{0}\right\|_{H^{s+2}}+\left\|u_{1}\right\|_{H^{s}}\right) \\
& +C \int_{0}^{\frac{t}{2}}(1+t-\tau)^{-\left(\frac{n}{4}+\frac{l}{2}+1\right)}\|f(u)\|_{L^{1}} d \tau \\
& +C \int_{\frac{t}{2}}^{t}(1+t-\tau)^{-\left(\frac{n}{4}+1\right)}\left\|\partial_{x}^{l} f(u)\right\|_{L^{1}} d \tau+C \int_{0}^{t} e^{-c(t-\tau)}\left\|\partial_{x}^{l+2} f(u)\right\|_{L^{2}} d \tau \\
\leq & C(1+t)^{-\left(\frac{n}{4}+\frac{l}{2}+1\right)}\left(\left\|u_{0}\right\|_{\dot{W}^{-1,1}}+\left\|u_{1}\right\|_{\dot{W}^{-2,1}}\right)+C e^{-c t}\left(\left\|u_{0}\right\|_{H^{s+2}}+\left\|u_{1}\right\|_{H^{s}}\right) \\
& +C \int_{0}^{\frac{t}{2}}(1+t-\tau)^{-\left(\frac{n}{4}+\frac{l}{2}+1\right)}\|u\|_{L^{2}}^{2} d \tau \\
& +C \int_{\frac{t}{2}}^{t}(1+t-\tau)^{-\left(\frac{n}{4}+1\right)}\|u\|_{L^{2}}\left\|\partial_{x}^{l} u\right\|_{L^{2}} d \tau \\
& +C \int_{0}^{t} e^{-c(t-\tau)}\left\|\partial_{x}^{l+2} u\right\|_{L^{2}}\|u\|_{L^{\infty}} d \tau \\
\leq & C(1+t)^{-\left(\frac{n}{4}+\frac{l}{2}+1\right)}\left(\left\|u_{0}\right\|_{\dot{W}^{-1,1}}+\left\|u_{1}\right\|_{\dot{W}^{-2,1}}\right)+C e^{-c t}\left(\left\|u_{0}\right\|_{H^{s+2}}+\left\|u_{1}\right\|_{H^{s}}\right) \\
& +C R^{2} \int_{0}^{\frac{t}{2}}(1+t-\tau)^{-\left(\frac{n}{4}+\frac{l}{2}+1\right)}(1+\tau)^{-\left(\frac{n}{2}+1\right)} d \tau \\
& +C R^{2} \int_{\frac{t}{2}}^{t}(1+t-\tau)^{-\left(\frac{n}{4}+1\right)}(1+\tau)^{-\left(\frac{n}{2}+\frac{l}{2}+1\right)} d \tau \\
& +C R^{2} \int_{0}^{t} e^{-c(t-\tau)}(1+\tau)^{-\left(\frac{n}{4}+\frac{l+2}{2}+\frac{1}{2}\right)}(1+\tau)^{-\left(\frac{n}{2}+\frac{1}{2}\right)} d \tau \\
\leq & C(1+t)^{-\left(\frac{n}{4}+\frac{l}{2}+1\right)}\left\{\left(\left\|u_{0}\right\|_{\dot{W}^{-1,1}}+\left\|u_{1}\right\|_{\dot{W}^{-2,1}}+\left\|u_{0}\right\|_{H^{s+2}}+\left\|u_{1}\right\|_{H^{s}}\right)+R^{2}\right\} .
\end{aligned}
$$

Thus

$$
(1+t)^{\frac{n}{4}+\frac{l}{2}+1}\left\|\partial_{x}^{l} \mathbb{T}(u)_{t}\right\|_{L^{2}} \leq C E_{0}+C R^{2} .
$$

Combining (4.5), (4.7) and taking $E_{0}$ and $R$ suitably small yields

$$
\|\mathbb{T}(u)\|_{X} \leq R .
$$


For $\tilde{u}, \bar{u} \in X_{R}$, by using (4.4), we have

$$
\mathbb{T}(\tilde{u})-\mathbb{T}(\bar{u})=\int_{0}^{t} G(t-\tau) * \Delta[f(\tilde{u})-f(\bar{u})] d \tau .
$$

Thanks to (4.9), (3.13) and Lemma 4.1, (4.1), for $k \leq s+2$, we obtain

$$
\begin{aligned}
& \left\|\partial_{x}^{k}(\mathbb{T}(\tilde{u})-\mathbb{T}(\bar{u}))\right\|_{L^{2}} \leq \int_{0}^{t}\left\|\partial_{x}^{k} G(t-\tau) * \Delta[f(\tilde{u})-f(\bar{u})]\right\|_{L^{2}} d \tau \\
& \leq C \int_{0}^{\frac{t}{2}}(1+t-\tau)^{-\left(\frac{n}{4}+\frac{k}{2}+\frac{1}{2}\right)}\|(f(\tilde{u})-f(\bar{u}))\|_{L^{1}} d \tau \\
& +C \int_{\frac{t}{2}}^{t}(1+t-\tau)^{-\left(\frac{n}{4}+\frac{1}{2}\right)}\left\|\partial_{x}^{k}(f(\tilde{u})-f(\bar{u}))\right\|_{L^{1}} d \tau \\
& +C \int_{0}^{t} e^{-c(t-\tau)}\left\|\partial_{x}^{k}(f(\tilde{u})-f(\bar{u}))\right\|_{L^{2}} d \tau \\
& \leq C \int_{0}^{\frac{t}{2}}(1+t-\tau)^{-\left(\frac{n}{4}+\frac{k}{2}+\frac{1}{2}\right)}\left(\|\tilde{u}\|_{L^{2}}+\|\bar{u}\|_{L^{2}}\right)\|\tilde{u}-\bar{u}\|_{L^{2}} \\
& +C \int_{\frac{t}{2}}^{t}(1+t-\tau)^{-\left(\frac{n}{4}+\frac{1}{2}\right)}\left\{\left(\left\|\partial_{x}^{k} \tilde{u}\right\|_{L^{2}}+\left\|\partial_{x}^{k} \tilde{u}\right\|_{L^{2}}\right)\|\tilde{u}-\bar{u}\|_{L^{2}}\right. \\
& \left.+\left(\|\tilde{u}\|_{L^{2}}+\|\bar{u}\|_{L^{2}}\right)\left\|\partial_{x}^{k}(\tilde{u}-\bar{u})\right\|_{L^{2}}\right\} d \tau \\
& +C \int_{0}^{t} e^{-c(t-\tau)}\left\{\left(\left\|\partial_{x}^{k} \tilde{u}\right\|_{L^{2}}+\left\|\partial_{x}^{k} \tilde{u}\right\|_{L^{2}}\right)\|\tilde{u}-\bar{u}\|_{L^{\infty}}\right. \\
& \left.+\left(\|\tilde{u}\|_{L^{\infty}}+\|\bar{u}\|_{L^{\infty}}\right)\left\|\partial_{x}^{k}(\tilde{u}-\bar{u})\right\|_{L^{2}}\right\} d \tau \\
& \leq C R\|\tilde{u}-\bar{u}\|_{X} \int_{0}^{\frac{t}{2}}(1+t-\tau)^{-\left(\frac{n}{4}+\frac{k}{2}+\frac{1}{2}\right)}(1+\tau)^{-\left(\frac{n}{2}+1\right)} d \tau \\
& +C R\|\tilde{u}-\bar{u}\|_{X} \int_{\frac{t}{2}}^{t}(1+t-\tau)^{-\left(\frac{n}{4}+\frac{1}{2}\right)}(1+\tau)^{-\left(\frac{n}{2}+\frac{k}{2}+1\right)} d \tau \\
& +C C R\|\tilde{u}-\bar{u}\|_{X} \int_{0}^{t} e^{-c(t-\tau)}(1+\tau)^{-\left(\frac{3 n}{4}+\frac{k}{2}+1\right)} d \tau \\
& \leq C R(1+t)^{-\left(\frac{n}{4}+\frac{k}{2}+\frac{1}{2}\right)}\|\tilde{u}-\bar{u}\|_{X},
\end{aligned}
$$

which implies

$$
(1+t)^{\frac{n}{4}+\frac{k}{2}+\frac{1}{2}}\left\|\partial_{x}^{k}(\mathbb{T}(\tilde{u})-\mathbb{T}(\bar{u}))\right\|_{L^{2}} \leq C R\|\tilde{u}-\bar{u}\|_{X} .
$$

Similarly, for $l \leq s$, from (4.6), (3.14) and (4.1), we have

$$
\begin{aligned}
\left\|\partial_{x}^{l}(\mathbb{T}(\tilde{u})-\mathbb{T}(\bar{u}))_{t}\right\|_{L^{2}} \leq & \int_{0}^{t}\left\|\partial_{x}^{l} G_{t}(t-\tau) * \Delta[f(\tilde{u})-f(\bar{u})]\right\|_{L^{2}} d \tau \\
\leq & C \int_{0}^{\frac{t}{2}}(1+t-\tau)^{-\frac{n}{4}-\frac{l}{2}-1}\|(f(\tilde{u})-f(\bar{u}))\|_{L^{1}} d \tau \\
& +C \int_{\frac{t}{2}}^{t}(1+t-\tau)^{-\frac{n}{4}-1}\left\|\partial_{x}^{l}(f(\tilde{u})-f(\bar{u}))\right\|_{L^{1}} d \tau
\end{aligned}
$$




$$
\begin{aligned}
& +C \int_{0}^{t} e^{-c(t-\tau)}\left\|\partial_{x}^{l+2}(f(\tilde{u})-f(\bar{u}))\right\|_{L^{2}} d \tau \\
\leq & C R(1+t)^{-\left(\frac{n}{4}+\frac{l}{2}+1\right)}\|\tilde{u}-\bar{u}\|_{X},
\end{aligned}
$$

which implies

$$
(1+t)^{\frac{n}{4}+\frac{l}{2}+1}\left\|\partial_{x}^{l}(\mathbb{T}(\tilde{u})-\mathbb{T}(\bar{u}))_{t}\right\|_{L^{2}} \leq C R\|\tilde{u}-\bar{u}\|_{X}
$$

Combining (4.10), (4.11) and taking $R$ suitably small yields

$$
\|\mathbb{T}(\tilde{u})-\mathbb{T}(\bar{u})\|_{X} \leq \frac{1}{2}\|\tilde{u}-\bar{u}\|_{X}
$$

From (4.8) and (4.12), we know that $\mathbb{T}$ is a strictly contracting mapping. Consequently, we conclude that there exists a fixed point $u \in X_{R}$ of the mapping $\mathbb{T}$, which is a classical solution to (1.1), (1.2). We have completed the proof of the theorem.

\section{Competing interests}

The author declares that she has no competing interests.

\section{Author's contributions}

The author completed the paper by herself. The author read and approved the final manuscript.

\section{Acknowledgements}

This work was supported in part by the NNSF of China (Grant No. 11101144).

Received: 13 April 2013 Accepted: 4 July 2013 Published: 16 July 2013

\section{References}

1. Boussinesq, J: Théorie des ondes et des remous qui se propagent le long dùn canal rectangulaire horizontal, en communiquant au liquide contenu dans ce canal des vitesses sensiblement pareilles de la surface au fond. J. Math. Pures Appl. 17, 55-108 (1872)

2. Bona, JL, Sachs, RL: Global existence of smooth solutions and stability of solitary waves for a generalized Boussinesq equation. Commun. Math. Phys. 118, 15-29 (1988)

3. Liu, Y: Decay and scattering of small solutions of a generalized Boussinesq equation. J. Funct. Anal. 147, 51-68 (1997)

4. Cho, Y, Ozawa, T: On small amplitude solutions to the generalized Boussinesq equations. Discrete Contin. Dyn. Syst. 17, 691-711 (2007)

5. Farah, L, Wang, H: Global solutions in lower order Sobolev spaces for the generalized Boussinesq equation. Electron. J. Differ. Equ. 2012(41), 1-13 (2012)

6. Varlamov, W: On the Cauchy problem for the damped Boussinesq equation. Differ. Integral Equ. 9, 619-634 (1996)

7. Varlamov, W: Existence and uniqueness of a solution to the Cauchy problem for the damped Boussinesq equation. Math. Methods Appl. Sci. 19, 639-649 (1996)

8. Varlamov, W: Asymptotic behavior of solutions of the damped Boussinesq equation in two space dimensions. Int. J. Math. Math. Sci. 22, 131-145 (1999)

9. Dai, W-R, Kong, D-X: Global existence and asymptotic behavior of classical solutions of quasilinear hyperbolic systems with linearly degenerate characteristic fields. J. Differ. Equ. 235, 127-165 (2007)

10. Kong, D-X, Yang, T: Asymptotic behavior of global classical solutions of quasilinear hyperbolic systems. Commun. Partial Differ. Equ. 28, 1203-1220 (2003)

11. Nakao, M, Ono, K: Existence of global solutions to the Cauchy problem for the semilinear dissipative wave equations. Math. Z. 214, 325-342 (1993)

12. Nishihara, $K: L^{p}-L^{q}$ estimates of solutions to the damped wave equation in 3-dimensional space and their applications. Math. Z. 244, 631-649 (2003)

13. Ono, K: Global existence and asymptotic behavior of small solutions for semilinear dissipative wave equations. Discrete Contin. Dyn. Syst. 9, 651-662 (2003)

14. Wang, W, Wang, W: The pointwise estimates of solutions for semilinear dissipative wave equation in multi-dimensions. J. Math. Anal. Appl. 368, 226-241 (2010)

15. Yang, Z: Longtime behavior of the Kirchhoff type equation with strong damping on $\mathbb{R}^{n}$. J. Differ. Equ. 242, 269-286 (2007)

16. Sugitani, Y, Kawashima, S: Decay estimates of solution to a semi-linear dissipative plate equation. J. Hyperbolic Differ. Equ. 7, 471-501 (2010)

17. Wang, Y-X: Existence and asymptotic behaviour of solutions to the generalized damped Boussinesq equation Electron. J. Differ. Equ. 2012(96), 1-11 (2012) 
18. Wang, Y-Z, Liu, FG, Zhang, YZ: Global existence and asymptotic of solutions for a semi-linear wave equation. J. Math. Anal. Appl. 385, 836-853 (2012)

19. Wang, $\mathrm{S}, \mathrm{Xu}, \mathrm{H}$ : On the asymptotic behavior of solution for the generalized IBq equation with hydrodynamical damped term. J. Differ. Equ. 252, 4243-4258 (2012)

20. Li, TT, Chen, YM: Nonlinear Evolution Equations. Scientific Press, Beijing (1989) (in Chinese)

21. Zheng, SM: Nonlinear Evolution Equations. Monographs and Surveys in Pure and Applied Mathematics, vol. 133. Chapman Hall/CRC, Boca Raton (2004)

doi:10.1186/1029-242X-2013-323

Cite this article as: Wang: Asymptotic decay estimate of solutions to the generalized damped Bq equation. Journal of Inequalities and Applications 2013 2013:323.

Submit your manuscript to a SpringerOpen ${ }^{\circ}$ journal and benefit from:

- Convenient online submission

- Rigorous peer review

- Immediate publication on acceptance

- Open access: articles freely available online

- High visibility within the field

- Retaining the copyright to your article

Submit your next manuscript at $>$ springeropen.com 